\title{
Précis zu The Normativity of Rationality
}

Benjamin Kiesewetter

Erscheint in: Zeitschrift für philosophische Forschung 71(4): 560-4 (2017).

Manchmal sind wir irrational. Der eine ist willensschwach: Er glaubt, dass er mit dem Rauchen aufhören sollte, bildet aber nicht die Absicht aus, dies auch zu tun. Der nächste beabsichtigt zwar, mit dem Rauchen aufzuhören, ergreift dann aber nicht die Mittel, die er zu diesem Zweck für notwendig erachtet. Ein Dritter hat inkonsistente Überzeugungen: er glaubt an die Evolutionstheorie, und glaubt auch, dass die Evolutionstheorie mit der Bibel nicht vereinbar ist, glaubt aber dennoch weiter an die Bibel. Ist daran eigentlich etwas auszusetzen? Sollten wir rational sein und Irrationalität vermeiden? Dies ist die Frage, um die es in meinem Buch geht.

Die intuitive Antwort auf diese Frage lautet ,ja“: Wenn wir eine Person als irrational bezeichnen, kritisieren wir sie, und damit sagen wir auch, dass ihr Zustand der Überprüfung und Änderung bedarf. Dies ist jedenfalls die These, die ich im zweiten Kapitel des Buchs (das auf eine allgemeine Einleitung im ersten Kapitel folgt) verteidige:

(T1) Gewöhnliche Zuschreibungen von Irrationalität sind Ausdruck von Kritik und setzen deshalb die Normativität der Rationalität voraus.

In meinem Buch verteidige ich diese Voraussetzung gegen Positionen, die sich dazu gezwungen sehen, die Normativität der Rationalität zurückzuweisen, und damit auf eine Fehlertheorie über gewöhnliche Irrationalitätszuschreibungen festgelegt sind.

Ich möchte damit beginnen, kurz einige Begriffe und Positionen einführend zu erläutern, die für die Darstellung meiner Argumentation wichtig sind. Der Begriff der Irrationalität setzt die Idee voraus, dass Lebewesen, die mit rationalen Fähigkeiten ausgestattet sind, bestimmten Standards der Rationalität unterliegen, die sie verletzen können und deren Verletzung ein Rationalitätsdefizit konstituiert. Die Frage nach der Normativität der Rationalität verstehe ich 
als die Frage, ob wir ausschlaggebende Gründe haben, die Standards der Rationalität zu erfüllen.

Ein Rationalitätsdefizit kann schlicht darin bestehen, dass wir nicht korrekt auf Gründe reagieren, die uns zur Verfügung stehen. Zum Beispiel können wir nicht als vollkommen rational gelten, wenn wir Überzeugungen haben, die durch unsere Evidenz nicht hinreichend gestützt werden. Entsprechende Rationalitätsstandards sind in einem trivialen Sinne durch normative Gründe gestützt. Virulent wird die Frage der Normativität, wenn wir uns dem Phänomen struktureller Irrationalität zuwenden. Dabei handelt es sich um eine Form der Irrationalität, die wir feststellen können, indem wir nur auf die Kombination verschiedener mentaler Einstellungen einer Person schauen und von den Gründen, die diese Person für die jeweiligen Einstellungen hat, abstrahieren. Die eingangs genannten Fälle von inkonsisten Überzeugungen oder Zweck/Mittel-Einstellungen sind hierfür beispielhaft. Im Buch konzentriere ich mich auf vier paradigmatische Fälle struktureller Irrationalität, die ich hier in leicht vereinfachter Form wiedergebe:

\section{Beispiele für strukturelle Irrationalität}

(AI) Akratische Irrationalität: Wenn A glaubt, dass sie $\phi$-en sollte, und A nicht beabsichtigt zu $\phi$-en, dann ist A irrational.

(II) Instrumentelle Irrationalität: Wenn A beabsichtigt $\mathrm{zu} \phi$-en, und A $\psi$-en für ein notwendiges Mittel hält, und A nicht beabsichtigt zu $\psi$-en, dann ist A irrational.

(DI) Doxastische akratische Irrationalität: Wenn A glaubt, dass sie hinreichende Evidenz für $\mathrm{p}$ hat, und A nicht glaubt, dass $\mathrm{p}$, dann ist $\mathrm{A}$ irrational.

(MI) Modus ponens-Irrationalität: Wenn A glaubt, dass $\mathrm{p}$, und A glaubt, dass $\mathrm{p} \rightarrow \mathrm{q}$, und A nicht glaubt, dass q, dann ist A irrational.

Diese Aussagen (AI)-(MI) geben hinreichende Bedingungen für Irrationalität an, die rein strukturell sind, d.h. unabhängig von den Gründen bestehen, die Akteure jeweils für ihre Einstellungen haben. So besagt z.B. Aussage (AI), dass eine Akteurin, die glaubt, dass sie alles in allem $\phi$-en sollte, nicht gleichzeitig rationalerweise von der Absicht zu $\phi$-en Abstand nehmen kann. Dies gilt offenbar unabhängig davon, ob sie gute Gründe für ihren Glauben oder die entsprechende Absicht hat oder nicht. 
Wenn nun solche rein strukturellen Bedingungen hinreichend für Irrationalität sind, und wenn Irrationalität die Verletzung eines Rationalitätsstandards impliziert, dann scheint es sehr nahe liegend anzunehmen (wie das in der gegenwärtigen Forschungsliteratur auch getan wird), dass es genuin strukturelle Rationalitätsstandards gibt - etwa einen Standard, der von uns fordert, nicht zu glauben, dass wir etwas tun sollen, ohne es dabei auch zu beabsichtigen.

Vor diesem Hintergrund ergibt sich ein philosophisches Problem, das sich als ein Trilemma beschreiben lässt. Erstens scheint es aufgrund der genannten Erwägungen natürlich, davon auszugehen, dass es strukturelle Rationalitätsstandards gibt. Zweitens legt, wie These (T1) besagt, unsere Praxis der Zuschreibung von Irrationalität nahe, dass Rationalitätsstandards normativ verbindlich sind. Drittens jedoch scheinen eine Reihe von plausiblen Argumenten in der aktuellen Debatte zu zeigen, dass strukturelle Rationalitätsstandards nicht normativ sein können. Die genannten drei Aussagen sind jedoch logisch inkompatibel:

(i) Es gibt strukturelle Rationalitätsstandards.

(ii) Rationalitätsstandards sind normativ.

(iii) Strukturelle Rationalitätsstandards sind, sofern es sie gibt, nicht normativ.

Entsprechend müssen wir eine dieser Aussagen aufgeben. Daraus ergeben sich drei theoretische Optionen:

$\neg$ (iii) Die strukturalistische Auffassung: Es gibt strukturelle Rationalitätsstandards, und diese sind auch normativ.

$\neg$ (ii) Die gemischte Auffassung: Es gibt strukturelle Rationalitätsstandards, aber diese sind nicht normativ.

$\neg$ (i) Die anti-strukturalistische Auffassung: Es gibt keine strukturellen Rationalitätsstandards.

Die strukturalistische Auffassung behauptet die Existenz normativer struktureller Rationalitätsstandards. Wie ich in den Kapiteln 3-5 des Buches ausführlich darlege, scheitert diese Auffassung jedoch:

(T2) Die Annahme, dass wir normative Gründe haben, strukturelle Rationalitätsstandards zu erfüllen, ist unüberbrückbaren Schwierigkeiten ausgesetzt. 
Eine dieser Schwierigkeiten ist das sogenannte Bootstrapping-Problem: So scheint etwa ein normativ verstandener Rationalitätsstandard, der akrasia verbietet, darauf hinauszulaufen, dass wir immer ausschlaggebenden Grund haben, das zu beabsichtigen, von dem wir faktisch glauben, dass wir es tun sollen. Dies jedoch ist absurd. Nur weil jemand glaubt, dass er z.B. Donald Trump wählen sollte, folgt noch lange nicht, dass er auch guten Grund hat, dies zu beabsichtigen.

Nahezu alle Philosophen, die die in These (T2) zum Ausdruck kommende Ansicht teilen, haben daraus die Konsequenz gezogen, die Normativität der Rationalität aufzugeben, und stattdessen diejenige Sichtweise anzunehmen, die ich im Buch als die ,gemischte Auffassung“ bezeichne. Nach dieser Auffassung gibt es zwar strukturelle Rationalitätsstandards, aber diese sind nicht normativ. Ziel meiner Arbeit ist es, die strukturalistische und die gemischte Auffassung zugunsten einer dritten, anti-strukturalistischen Auffassung zurückzuweisen, die die Normativität rationaler Standards verteidigt, indem sie die Existenz struktureller Rationalitätsstandards zurückweist.

Die ersten beiden der genannten Thesen legen diese Sichtweise bereits nahe. In Kapitel 6 argumentiere ich darüber hinaus gehend:

(T3) Die Annahme struktureller Rationalitätsstandards ist ganz unabhängig von der Frage ihrer Normativität unhaltbar.

Dieses Kapitel schließt den negativen Teil des Buches ab, in welchem die strukturalistische und die gemischte Auffassung zurückgewiesen werden. Die anti-strukturalistische Auffassung ergibt sich als logische Konsequenz. Ihre Ausarbeitung und Substantiierung, der ich mich im zweiten Teil des Buches widme, steht jedoch vor zwei wichtigen Herausforderungen.

Erstens muss eine alternative, nicht-strukturalistische Rationalitätskonzeption entwickelt werden, die mit der Normativität der Rationalität vereinbar ist. Zweitens bedarf es einer Erklärung, warum strukturelle Bedingungen hinreichend für Irrationalität sein können, wenn es keine strukturellen Rationalitätsstandards gibt. Meine Antwort auf die erste dieser beiden Herausforderungen gebe ich in Kapitel 7:

(T4) Eine plausible alternative Rationalitätskonzeption setzt bei dem Gedanken an, dass Rationalität von uns erfordert, auf Gründe korrekt zu reagieren, die uns epistemisch zur Verfügung stehen (d.h. Teil unserer Evidenz sind). 
Die sich aus dieser Konzeption ergebenden Gründe-Reaktions-Standards der Rationalität sind notwendigerweise normativ in dem Sinne, dass es eine signifikante Gruppe von Gründen (nämlich die der epistemisch zugänglichen Gründe) gibt, die für die Einhaltung dieser Standards sprechen. Es lässt sich jedoch darüber hinaus zeigen:

(T5) Was eine Person alles in allem tun soll (ausschlaggebenden Grund hat zu tun) hängt ausschließlich von Gründen ab, die ihr epistemisch zur Verfügung stehen.

Wie ich in Kapitel 8 argumentiere, wird nur eine solche evidenz-relative Konzeption von Gründen der Tatsache gerecht, dass wir unter Bedingungen von Unsicherheit verantwortliche Entscheidungen treffen können. Es folgt, dass Rationalitätsstandards, die eine korrekte Reaktion auf epistemisch zugängliche Gründe erfordern, im stärksten möglichen Sinne normativ sind: sie gehen notwendigerweise mit ausschlaggebenden Gründen einher.

Die verbleibende Aufgabe meiner Theorie ist es, das Phänomen der strukturellen Irrationalität zu erklären, ohne dabei auf strukturelle Rationalitätsstandards zurückzugreifen. Dieser Herausforderung widme ich mich, indem ich zuerst zwei nicht-strukturelle Rationalitätsstandards aus der Gründe-Reaktions-Konzeption der Rationalität ableite (vgl. Kapitel 7):

(T6) Die Gründe-Reaktions-Konzeption von Rationalität rechtfertigt die Annahme folgender beider nicht-struktureller Rationalitätsstandards:

TR Theoretische Rationalität: Hat A hinreichende Evidenz für $\mathrm{p},[\ldots]$ dann erfordert Rationalität von A, zu glauben, dass p. Hat A keine hinreichende Evidenz für p, dann erfordert Rationalität von A, nicht zu glauben, dass p.

PR Praktische Rationalität: Hat A hinreichende Evidenz dafür, dass sie $\phi$-en sollte, [...] dann erfordert Rationalität von A, $\phi$-en $\mathrm{zu}$ beabsichtigen. Hat $\mathrm{A}$ hinreichende Evidenz dafür, dass sie nicht $\phi$-en sollte, dann erfordert Rationalität von A, $\phi$-en nicht zu beabsichtigen.

In den Kapiteln 9 und 10 versuche ich schließlich zu zeigen: 
(T7) Der Umstand, dass rein strukturelle Bedingungen hinreichend für Irrationalität sein können, kann allein durch Rekurs auf nicht-strukturelle Rationalitätsstandards wie (TR) und (PR) erklärt werden.

Diese These sei hier kurz und in vereinfachter Weise am Beispiel der akratischen Irrationalität illustriert:

Beispiel: Akrasia

1. Entweder A hat hinreichende Evidenz dafür, dass sie $\phi$-en sollte, oder nicht (logische Wahrheit).

2. Wenn A diese Evidenz nicht hat, dann erfordert Rationalität von A, nicht zu glauben, dass sie $\phi$-en sollte (TR).

3. Wenn A diese Evidenz hat, dann erfordert Rationalität von A, $\phi$-en zu beabsichtigen $(P R)$.

4. Also: Entweder erfordert Rationalität von A, nicht zu glauben, dass sie $\phi$-en sollte, oder Rationalität erfordert von A, $\phi$-en zu beabsichtigen (aus 1, 2, und 3).

Dies erklärt: Wenn A glaubt, dass sie $\phi$-en sollte, und A nicht beabsichtigt zu $\phi$-en, dann ist A irrational (AI).

Wie dieses Argument illustriert, kann das Phänomen der strukturellen Irrationalität auch ohne die Annahme struktureller Standards der Rationalität erklärt werden - nicht-strukturelle Gründe-Reaktions-Standards wie TR und PR reichen dafür aus. Da die Annahme struktureller Standards ohnehin große Schwierigkeiten aufwirft, ist diese Erklärung vorzuziehen. Sie hat den weiteren Vorteil, dass sie der vortheoretischen Intuition gerecht werden kann, dass Irrationalität etwas Kritisierenswertes ist, und uns so die Möglichkeit eröffnet, die Frage, ob wir ausschlaggebende Gründe dafür haben, rational zu sein, zu bejahen.

\section{Literatur}

Kiesewetter, Benjamin (2017): The Normativity of Rationality. Oxford: Oxford University Press. 